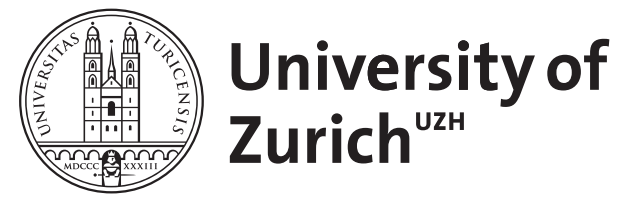

\title{
Use Cases as Means to support the Appropriation of Enterprise Social Software
}

\author{
Herzog, Christian ; Richter, Alexander
}

DOI: https://doi.org/10.1109/HICSS.2016.505

Posted at the Zurich Open Repository and Archive, University of Zurich ZORA URL: https://doi.org/10.5167/uzh-120508

Conference or Workshop Item

Originally published at:

Herzog, Christian; Richter, Alexander (2016). Use Cases as Means to support the Appropriation of Enterprise Social Software. In: 2016 Hawaii International Conference on System Sciences, Kauai, 5 January 2016 - 8 January 2016, IEEE.

DOI: https://doi.org/10.1109/HICSS.2016.505 


\section{Use Cases as a Means to Support the Appropriation of Enterprise Social Software}

\author{
Christian Herzog \\ University of Osnabrück \\ christian.herzog@uni-osnabrueck.de
}

\author{
Alexander Richter \\ University of Zurich \\ arichter@ifi.uzh.ch
}

\begin{abstract}
Despite the growing use of enterprise social software (ESS) to support internal communication or knowledge management, its successful introduction is still a challenge. Studies have shown that ESS needs to be appropriated by the employees by making sense of the tools and reflecting on its possible benefits for individual working practices. To support and accelerate this complex procedure and reduce the risk of non-appropriation, we propose a nascent design theory for use cases as a means to support ESS appropriation. Interviews with 10 department heads, usage observations, and a survey at a medium-sized German service provider serve as a database for applying a causal analysis, as suggested by Gregor et al. 2013. Our study provides structured guidance on how to conceptualize and utilize use cases to promote desired ESS uses and ultimately contributes to a better understanding of ESS appropriation.
\end{abstract}

\section{Introduction}

The constantly rising popularity of social platforms such as Facebook and Twitter has led many companies to explore the potential of such platforms to support their internal collaborative work practices [1, 15]. Whereas the capability to use these tools is slowly maturing [25], companies are increasingly realizing the advantages of using enterprise social software (ESS), such as better knowledge sharing and enhanced employee innovativeness $[5,8,12]$.

While science still finds it difficult to draw the line between ESS and other information systems (IS) (cf. Section 5.2), recent studies argue that existing theories and methods have to be revisited and probably adjusted to explain the specific phenomena associated with ESS $[1,25]$. In this context, while there is a growing number of studies explicating user behavior in ESS [2, $6,15]$, there is still a lack of understanding how such platforms can be introduced.

ESS is facing plenty of obstacles before it will finally be adopted in daily business life [33]. For example, there are often doubts that the tools would bring the predicted benefit [27], and the software features are in competition with proven ways of communication, such as e-mail. Related concerns are that the ESS may therefore be rejected [35] and that the company-wide ESS adoption depends on users' awareness of the potential use benefits [32].

The reason for this is that, compared with other kinds of IS, e.g. ERP systems, ESS can be characterized as malleable end-user software, i.e. it does not lend itself to immediate forms of usage determined or prescribed by its features [37]. Instead, the users have to explore the potential of ESS over time. Against this backdrop, the emergence of ESS potentially requires means to support its appropriation [35].

We conducted a long-term exploratory case study with a leading, medium-sized German service provider for digital corporate communications. By interviewing 10 heads of department, making usage observations and carrying out a survey with 58 participants, we focused on the following research question:

How can use cases be designed in order to support ESS appropriation?

To answer this question we follow the design theory approach of Gregor et al. 2013 [20]. Based on creative causation, passive causation and active causation [20], we examine our dataset with respect to the purpose and scope, as well as the principles, of form and function. The outcome of this study is a nascent design theory as described by Gregor and Jones [19] that provides structured guidance on how to conceptualize and utilize use cases to communicate desired ESS uses. Our study supports the ongoing screening and examination of existing uses, helps users to orientate and converge their uses and ultimately contributes to a better understanding of ESS appropriation.

In the following section, we briefly describe the nascent design theory's justificatory knowledge, before we introduce our research methodology in greater detail. Next, we present the case study that served as a starting point and collected empirical data. Based on the findings of our causal analysis in Section 4, we 
explain the conceptual model, i.e. its purpose and its principles of form and function. We conclude with a brief summary and an outlook on further research.

\section{Justificatory knowledge}

Over the past few years, ESS has made fast inroads into organizations. Practitioner reports, as well as scientific works, have revealed how ESS can improve information sharing, communication and group work within a company $[1,5]$. Nevertheless, studies have also shown that the abovementioned benefits can only be achieved if a majority of the users adopt the ESS for their daily business tasks $[11,23]$. Related works point to a variety of factors influencing the adoption of an ESS [8, 12, 25], and persons responsible for the introduction of an ESS are facing a number of challenges.

A reportedly major challenge is the lack of a clear purpose and guidelines for employees on how to use $\mathrm{ESS}$, since it is a malleable, social technology and turns out to be quite different in various contexts [37]. As such, ESS does not lend itself to immediate forms of usage determined or prescribed by their features, and therefore it is hard to predict how and in what form ESS will be put to use when rolled out in a particular organizational context [36].

This can be explained by existing and established theories like the concept of technologies-in-practice [30]. According to Orlikowski and Iacono [30], technologies when interpreted in the context of practices differ markedly, like team coordination or project updates. At the same time, ESS be conceptualized as an information infrastructure, i.e. as "a shared, open (and unbounded) heterogeneous and evolving socio-technical system (which we call installed base) consisting of a set of IT and their user, operations and design communities" [46, p. 748]. An information infrastructure does not have a predefined goal but may be used for various purposes and in various ways. Hence an information infrastructure needs to be interpreted and appropriated by its users and thus be integrated into existing work practices.

Dourish defines appropriation as the process by which the employees adopt and adapt technologies, fitting them into their daily working practices. It concerns the adoption patterns of technology and the transformation of practice at a deeper level [7]. The use of technology can also go beyond that, for what it was originally intended [7]. Especially with ESS it is difficult to predict how it is appropriated due to the malleability. The definition of use cases (cf. Section 5.1) appears in publications as a possible approach to overcome this problem [41]. However, studies about the specific design of use cases for the ESS appropriation are missing.

In conclusion, in order to be appropriated in a particular context, users need a better understanding of these technologies and their benefits for organizational practices. In the following we propose a nascent design theory that positions use cases as a means to communicate desired ESS uses in a way to guide employees and help them to orientate and converge their uses. Thus, it helps to provide a structured guidance on how to conceptualize and utilize these use cases. Next, we will explain our research approach in more detail.

\section{Research design}

Our paper is the outcome of a long-term exploratory case study [9, 13, 39] with the EQS Group AG (hereafter termed as "EQS"), a medium-sized provider for digital corporate communications and investor relations [14]. Part of the case study was an investigation of collaborative work practices.

After having captured a detailed view of work practices at EQS, we reflected on our findings in order to create a theory for the design of use cases for ESS appropriation [20]. Thus, we elaborated a set of more general guidelines that are applicable to a broader field [47]. The following sections present the case, the data set, and the research methodology in further detail.

\subsection{Case: EQS Group AG}

Our case company, the EQS Group AG, was founded in 2000 in Munich, Germany. EQS currently has over 170 employees in seven countries and more than 7,000 customers worldwide [14]. EQS's global distribution is a key factor in the acquisition of international customers but also implies the need of close worldwide collaboration between employees in different locations. EQS's portfolio consists mainly of web services and digital products such as corporate websites, apps, and news distribution or single website tools. The organizational culture can be described as co-operative, open, and little bureaucratic. Employees can organize their daily work in their own way, which leads to more flexibility.

At the same time, driven by the fast growth rate of the company, the absence of compulsory and established workflows also leads to an amount of individually varying collaborative work practices for the same purpose. For instance, there are several tools to communicate and explore innovative ideas within the company. This reduces communication barriers, but on the other hand the time for releasing new ideas 
is increased, as the innovation process is not streamlined. The use of ESS supports the collaboration and communications processes at EQS, while the work practices are mainly bundled there. These circumstances make EQS a suitable case for our study to investigate the appropriation support of ESS by defined use cases.

The investigated ESS subject that EQS uses is based on Atlassian's Confluence, a commercial wiki software with several socio-technological collaboration functions, including blogs, microblogs, social networking, etc. It was introduced in January 2013 with an iterative rollout, which means that the different departments have been trained and brought up to the ESS sequentially. Thus, on the one hand overcoming the critical mass is supported [26, 40], and on the other hand, first experiences could be transferred to other departments. The project team included representatives from each department, so that different points of view were involved in the design of the ESS, and simultaneously the heads of the department gave their commitments [38]. Although most of the employees were already using the ESS for their daily collaborative work practices shortly after its launch, it was important for management that the "late majority" and the "laggards" [34] also appropriate the platform as soon as possible. The company-wide use of Confluence from all employees is established as an important strategic objective by the management.

\subsection{EQS dataset}

The analysis for the creation of our design theory is based on a mixed methods approach [49] that includes four data sources [9]: (1) interviews with 10 heads of department, (2) employee survey with 58 participants, (3) usage observations and ESS usage statistics, as well as (4) further company information like project documents. The data was collected and processed in a team of two researchers who were continually in contact with the participants of the study and other EQS representatives. To strengthen the validity of our data, we consulted existing literature on ESS and other types of IS appropriation (cf. Section 2) to form a priori constructs [17]. This also enabled us to prepare the interview guide as well as the survey questionnaires [4].

The 10 semi-structured interviews were conducted in May and June 2013 and had an average duration of 30 minutes. During the interviews, conversation notes were taken. The interviewees were selected after a thorough examination of the organizational configuration at EQS. This qualitative approach allowed us to investigate collaborative work practices in general and to identify potential use cases that are suitable for an ESS application [44, 45]. Furthermore, this helped us to draw conclusions about the required design properties of ESS use cases. The interview guide consists of 26 questions about existing and potential collaborative work practices, process steps, and barriers.

The subsequent explorative survey [16] helped us to examine the adopted ESS use cases and to understand the general approach of the utilization. Additionally we were able to identify the need of further use cases or their modification. The survey took place seven months after the rollout of the ESS (July and August 2013) and was open to all EQS employees, who had the possibility to participate within 12 days. Thus, the users were able to gain first experiences and appropriate first use cases. In total, 58 employees participated in the survey: 51 participants used the German questionnaires, and seven the English version. The survey was anonymous and consists of 17 questions in total, concerning the private use of social software, the extent of the utilization of the ESS as well as personal attitude toward the platform. In addition, the employees were questioned about different use cases in order to gain an insight into the actual situation.

The collection of usage statistics after 17 months (as on May 31, 2015) gave us an additional long-term assessment of the ESS appropriation [48].

\subsection{Research methodology}

Our study can be classified as design science research $[22,29,31]$ aiming to develop an explanatory, practitioner-oriented design theory $[3,19,47]$ that solves an important business problem [21]. We started with literature research, as described in Section 2. The presented existing studies provided justificatory knowledge [19] and served as kernel theories [50] that helped us to ensure the study is framed correctly.

To answer the presented research question, the approach of Gregory et al. is particularly suitable [20]: Gregor et al. propose a framework for developing design theories through inductive processes of reflection and abstraction. In this context, reflection refers to the process of learning from past experiences, and abstraction describes the process of deriving generic features from observed instances of artifacts. In our context, the reflective judgment of the ESS utilization helps to identify essential conditions that are applicable to a broader class of problems [28]. The malleability of ESS implies that the user will explore and establish several use cases over time as they are not a priori defined [36]. Since users voluntarily make the ESS appropriation and the use case design happen without active intervention, we assume that the 
cognitions can be adopted to a design theory. Against this backdrop, an investigation of the past ESS utilization is ideal to derive knowledge for the active use case design that supports company-wide ESS appropriation.

According to Gregor et al. [20], design theorizing operates in an instance domain and an abstract domain [28], and design theory can be extracted from instances of artifacts through three intertwined types of causal analysis: creative causation, passive causation, and active causation [18]. This causal analysis helps us to identify (1) the purpose and scope, the (2) principles of form, and the (3) principles of function of our design theory.

First, creative causation helps to reflect on the purpose and scope by focusing back on the initial design ideas and requirements of the artifact. Referring to this, we identify the use cases and the general utilization of the ESS by the observation of the ESS and the user survey. Therefore we were able to create an understanding of the initial use case design by the users and the idea as well as the purpose behind them. The interviews with the heads of the department further allowed us to evaluate other collaborative work practices and their characteristics apart from the ESS, so that the fundamental ideas can also be considered in the design theory.

Second, passive causation helps to reflect on principles of form by focusing on an artifact's affordances, i.e. essential artifact properties that facilitate the performance of some action in a specific context [18]. The identification of the properties of the use cases, the kind of actions and the user groups involved were examined by the user survey as well as the ESS observation. Furthermore, the literature analysis supported the linkage between the justificatory knowledge and the use case design in order to achieve underlying purposes.

Third, active causation helps to reflect on principles of function by analyzing the way an artifact operates to initiate the trajectory of a change [20]. In that step we actively adopt the insights and design new use cases for comprehensive ESS appropriation. The ideas created by the initial interviews in the creative causation assisted the design process [20]. Furthermore, justificatory knowledge about use case design supports the design process. In this way, the artifacts' mutability $[19,43]$ is proved. With regard to our case company, expository instantiations $[18,21]$ in the form of five example use cases are presented.

To make the analysis more transparent [9], we list the reflective questions and summarize the research methodology for our design theory in Figure 1. By considering past experiences and applying an iterative procedure of the use case designs, our approach can be classified as heuristic theorizing [21].

\section{Results}

In this section we present how we applied the causal analysis to extract a design theory from our dataset.

For our creative causal analysis it was important to understand which ESS use cases had already been

\begin{tabular}{|c|c|c|c|}
\hline $\begin{array}{l}\text { Design theory } \\
\text { component }\end{array}$ & Abstraction process & Reflective questions & Method \\
\hline $\begin{array}{l}\text { 1. Purpose and } \\
\text { Scope }\end{array}$ & $\begin{array}{l}\text { Creative causal analysis } \\
\text { Identification of use cases }\end{array}$ & $\begin{array}{l}\text { - What kind of use cases are already adopted by the users in the ESS? } \\
\text { - idea behind them? What is the purpose? } \\
\text { Are there other use cases that can be solved by an ESS? Do they } \\
\text { require further innovative design ideas? }\end{array}$ & $\begin{array}{ll}\cdot & \text { User survey } \\
\text { - } & \text { ESS observation } \\
\text { - } & \text { Interviews with } \\
& \text { department heads }\end{array}$ \\
\hline $\begin{array}{l}\text { 2. Principles of } \\
\text { Form }\end{array}$ & $\begin{array}{l}\text { Passive causal analysis } \\
\text { Identification of the } \\
\text { properties of successful } \\
\text { use cases }\end{array}$ & $\begin{array}{l}\text { - What are the properties of the use cases that enable them to achieve } \\
\text { the purposes of the users? } \\
\text { - Which contextual conditions are observed to enable the emergence } \\
\text { of the desired affordances? } \\
\text { - Which user groups perceive which functional affordances of the } \\
\text { artifact? } \\
\text { What justificatory knowledge provides support for the linking of the } \\
\text { use case design to the achievement of its goals? }\end{array}$ & $\begin{array}{ll}\text { - } & \text { User survey } \\
\text { - } & \text { ESS observation } \\
& \text { Literature analysis }\end{array}$ \\
\hline $\begin{array}{l}\text { 3. Principles of } \\
\text { Function }\end{array}$ & $\begin{array}{l}\text { Active causal analysis } \\
\text { Design of use cases }\end{array}$ & $\begin{array}{l}\text { - Which use cases can be transfered to the } \\
\text { to be designed to support the ESS appro } \\
\text { - In what order should the actions be und } \\
\text { Which actions are necessary (or most ne } \\
\text { desired outcomes? Why are they necessd } \\
\text { support from justificatory knowledge)? }\end{array}$ & $\begin{array}{l}\text { - Interviews with } \\
\text { department heads } \\
\text { Use case design } \\
\text { aproach } \\
\text { - Literature analysis }\end{array}$ \\
\hline
\end{tabular}

Figure 1: Research methodology for the creation of the design theory according to Gregor et al. [20] 
adopted. At the time of the survey $64 \%$ [ $n=37]$ of all participants already had logged in to the platform at least daily in order to read news or documentations or to search for work-related information. However, a much lower percentage of users actually contributed frequently to the platform by adding content to it. For instance, $19 \%[\mathrm{n}=11]$ had never created a blog post or a page. The investigation of the ESS utilization showed that most of the users were mainly using the platform passively (for reading, receiving information, etc.). Although in general a majority of the employees already used the ESS, the need to support the appropriation from all employees further was reflected and also requested by the user in an open text field of the survey (participant 30 (p.30): "For me, the concentrated knowledge is very useful for our daily work. However, the platform is not used by all or not all are sharing information.")

In addition to the passive use, several use cases could already be identified, such as "write documentations," "inform my colleagues," "document meetings," and "submit feature requests." In this context, the demand of guidelines for unification and structuring arose. Even if the majority [n=30] could cope without more regulations for the ESS use, there were cases of application in which guidelines in the form of use case definitions were needed (p.10: "My suggestion would be to provide regular training and examples of use cases."). It is evident that a healthy mediocrity amount of use cases should be specified so that there is still enough freedom for ESS use.

We found that use cases are particularly suitable, and information that was previously spread in different places (in e-mails, drives, file folders, etc.) can be centralized. $67 \%[\mathrm{n}=39]$ of the participants in the survey claimed that Confluence is the first place to save EQS-based information, and 78\% [ $\mathrm{n}=45]$ even affirmed the statement that the platform is the first place to search for EQS-related information. Furthermore, users stated that avoiding duplication or additional effort is an important reason for adopting use cases. An example of this is the organization and documentation of meetings. Prior to the introduction of the ESS, there was no common approach for the organization of meetings, and protocols were sent via e-mail, which eventually became unsustainable. Consequently, on the one hand, the interviews had previously identified these use cases as an application in the ESS. On the other hand, users have already started to manage their meetings autonomously in the ESS. However, the subsequent need for a uniform and company-wide use arose to circumvent a variety of different approaches (p.5: "In addition, many documented processes are not met adequately by the users.") and double work (p.2: "The platform brings a benefit for the company [...] through the sharing of documents or information, duplication of work can be avoided.").

In the passive causal analysis, we examined the properties and elements of the use cases. Each use case identifies at least one unique benefit for the individuals (p.26: "Through the platform, for example, I can organize my own work"), the team (p.5: "Our team worked out a very good documentation basis to look up") or the organization (p.20: "Through knowledge sharing and storage, the company is independent on the knowledge of individual employees"). At the same time, the objective is an important part of the use cases. Some $90 \%[\mathrm{n}=52]$ agreed with the statement that they are aware of the goals pursued with the platform. Especially when using different collaboration tools, it is important that the objectives are set clearly so that everybody is aware of when to use the ESS (p.34: "Meanwhile, there are several tools, such as Confluence, instant messaging, e-mail, file drives, etc. I think there are too many platforms next to each other.").

Furthermore, the description and definition of the participating users or actors, as well as the location where the ESS use cases take place, are additional components. Therefore, it is essential to define precisely which employees are involved and could benefit from the use case. For instance, it can be seen that for an international cooperation, the language should always be English (p.54: "There is too much German content, especially about products and other related information"). In that regard, we have found that even users who are involved only passively can benefit from the use cases (p.11: "As an international location, we have the opportunity to experience something personal about our German colleagues and their work as well as disclose something from our side.").

As mentioned above, we saw that a detailed sequence description of the workflow is significant to present a unified approach and understanding of the use case transparently.

By means of the active causal analysis, we have taken different actions to actively introduce ESS use cases at EQS. For this reason it was important to involve the users actively in the design process in order to obtain their commitment and simultaneously align the use cases for their needs. Nearly one-third [ $\mathrm{N}=17]$ indicated that they had not been adequately involved when the ESS was introduced.

Five use cases were preselected (cf. Table 1) based on the interviews. One initial criterion for the preselection of the use cases was the characteristics of the involved roles and users. Related to the passive causal analysis, it is preferable that all users benefit 
from the ESS use case, as everyone could be a stakeholder in the process. Hence, no use case was selected that would incorporate only a small, isolated group of employees. Additionally, we found that the use cases should profit from the sociotechnical features of the ESS. For instance, the possibility of commenting on the content in the ESS can be a surplus for the whole process. Another aspect was the network effect that results from the introduction of a use case with the ESS.

Table 1: Selected use cases at EQS

\begin{tabular}{|l|l|}
\hline Name & Short description \\
\hline $\begin{array}{l}\text { Personal } \\
\text { profiles }\end{array}$ & $\begin{array}{l}\text { Creating profile pages for each employee, } \\
\text { easing the process of the introduction of a } \\
\text { new colleague, and finding a colleague } \\
\text { with a specific competence. }\end{array}$ \\
\hline $\begin{array}{l}\text { Meeting } \\
\text { organization }\end{array}$ & $\begin{array}{l}\text { Organizing meetings via the ESS to } \\
\text { distribute the meeting agenda, assign } \\
\text { tasks to the attendees, and make a } \\
\text { protocol of the results of the meeting }\end{array}$ \\
\hline $\begin{array}{l}\text { Project } \\
\text { order } \\
\text { management }\end{array}$ & $\begin{array}{l}\text { Managing the project orders with the } \\
\text { platform to provide an overview of } \\
\text { running orders, arranging the various } \\
\text { stakeholders, and creating a central } \\
\text { information space for all projects. }\end{array}$ \\
\hline $\begin{array}{l}\text { Feature } \\
\text { requests }\end{array}$ & $\begin{array}{l}\text { Creating a space where all employees } \\
\text { can leave their feature requests for products } \\
\text { that the firm is offering. }\end{array}$ \\
\hline $\begin{array}{l}\text { Employee } \\
\text { suggestions }\end{array}$ & $\begin{array}{l}\text { Creating an open space where all } \\
\text { employees can hand in suggestions } \\
\text { concerning their work, internal processes } \\
\text { or other company-related issues. }\end{array}$ \\
\hline
\end{tabular}

To check whether the use cases are suitable to support the ESS appropriation, they have been taken up and verified in the employee survey. In relation to the aforementioned example of the "meeting organization" use case, $97 \%[n=56]$ of employees indicated that they would like to receive the agenda in advance to prepare themselves. Furthermore, 50\% $[\mathrm{n}=29]$ reported that tasks from meetings are sometimes forgotten. A further $12 \%[\mathrm{n}=7]$ stated that this happens often or very often. As $28 \%[n=16]$ already use the ESS to organize meetings, it can be interpreted that there is a lack of unified specifications (p.24: "The prior notification and the documentation of meetings take place too rarely on the platform.").

Prior to the introduction of the use cases, we created a textual description and a graphical workflow description in order to achieve transparency and a common understanding of the use cases (cf. Fig. 2). This way of depicting use cases made us aware of several advantages for the stakeholders. For example, while modeling a use case, critical events will be identified that were not visible before. An even more important benefit of this representation is that various new interrogations will arise, once it comes to the detailed definition of the single actions and their surroundings.

After the use cases were modeled, they were presented to the management and approved. Here, four of the five selected use cases were introduced (due to time constraints, the introduction of the "employee suggestions" use case was shifted). The introduction took place incrementally in cooperation with the heads of department and key users. It can be seen that one of the most important success factor is the integration into daily working practices. This is supported by Confluence in the form of the possibility to create different templates. For instance, a template for the use case meeting organization was created, in which the participants, the date, and the agenda could be deposited. When creating a new page, the users can simply choose the template as a draft.

Although there are different influence factors, the long-term usage statistics show that those spaces in which the use cases are introduced are among the most active (based on the number of page views, created items, and edits). For example, the space for "project order management" is the third-most active (out of 34). In the 34 open spaces, an average of 12,555 activities (view, edit, create) per month are tracked. In total there are 6,450 content items (pages, blogs, comments and status updates), and each month 235 more are added on average. Here, we did not consider spaces and pages that are restricted to specific users in the count. Overall, the company-wide appropriation of the ESS can be considered successful, and the platform is assessed by management as the most important communication and collaboration tool for the EQS.

\section{Design theory}

The design theory provides a deeper understanding of the concept of use cases and their influence on the ESS appropriation. The following sections lay out the different components of the design theory [20].

\subsection{Purpose and scope}

Following Gregor and Jones [19], a design theory's purpose and scope form not only the goal of and the need for a theoretical foundation but also the (meta-) requirements [50] and the differentiation of application areas [10].

With the creative causal analysis of our dataset we aimed to identify the common purpose behind the use case artifact and its scope. Based on the interviews and usage observations, the requirements were specified 
further. The purpose of the proposed design theory for ESS use cases can be summarized as follows:

1. The design theory formulates specific guidelines on how to design use cases that aim to support the ESS appropriation

2. The use cases illustrate how daily work practices can be carried out with the help of ESS and make the benefits of ESS use clear

3. The use cases are supposed to converge work practices and to avoid the use of different tools for the same practice.

Our design theory is built on experiences of existing work practices in order to provide guidance on how to create and utilize use cases to support the appropriation of an ESS. As meta-requirement [50], the design theory claims to work for any kind of ESS use. Thereby, it must be distinguished between prescribed, designed use cases by external parties, the platform owners or the management and use cases designed and adopted by the users.

This paper focuses on ESS (as an IS type), which includes all social software tools and platforms used for the company internal corporate communication and collaboration and therefore requires skills in the area of computer-supported cooperative work [26]. Compendiously ESS is a web-based technology that support users' contributions of persistent objects to a shared pool and that enable company-wide responses to these objects. ESS comprises functionalities that visualize profile information and link users with one another (e.g. discover/subscribe/follow/friend).

A use case is a list of interactions between different objects in order to achieve an objective [24].

\subsection{Principles of form and function}

The following section presents the abstract 'blueprint' [19] with its construct entities and principles of form and function. Based on the justificatory knowledge and the presented case study, we provide a descriptive model for the design of ESS use cases. We aim to create a deeper understanding for the requirements by means of two components: a textual description and a graphical representation. Figure 2 depicts an expository instantiation [19, 22] of the "meeting organization" use case that we identified. Through the passive causal analysis we were able to identify the properties and constructs. The following five dimensions are an integral part of the textual description:

- Objective: Briefly describes what will be achieved with the use case. It should be as concrete as possible

- Benefit: Explains the benefits of this use case for the users, the team and the company. It demonstrates the alignment to general business

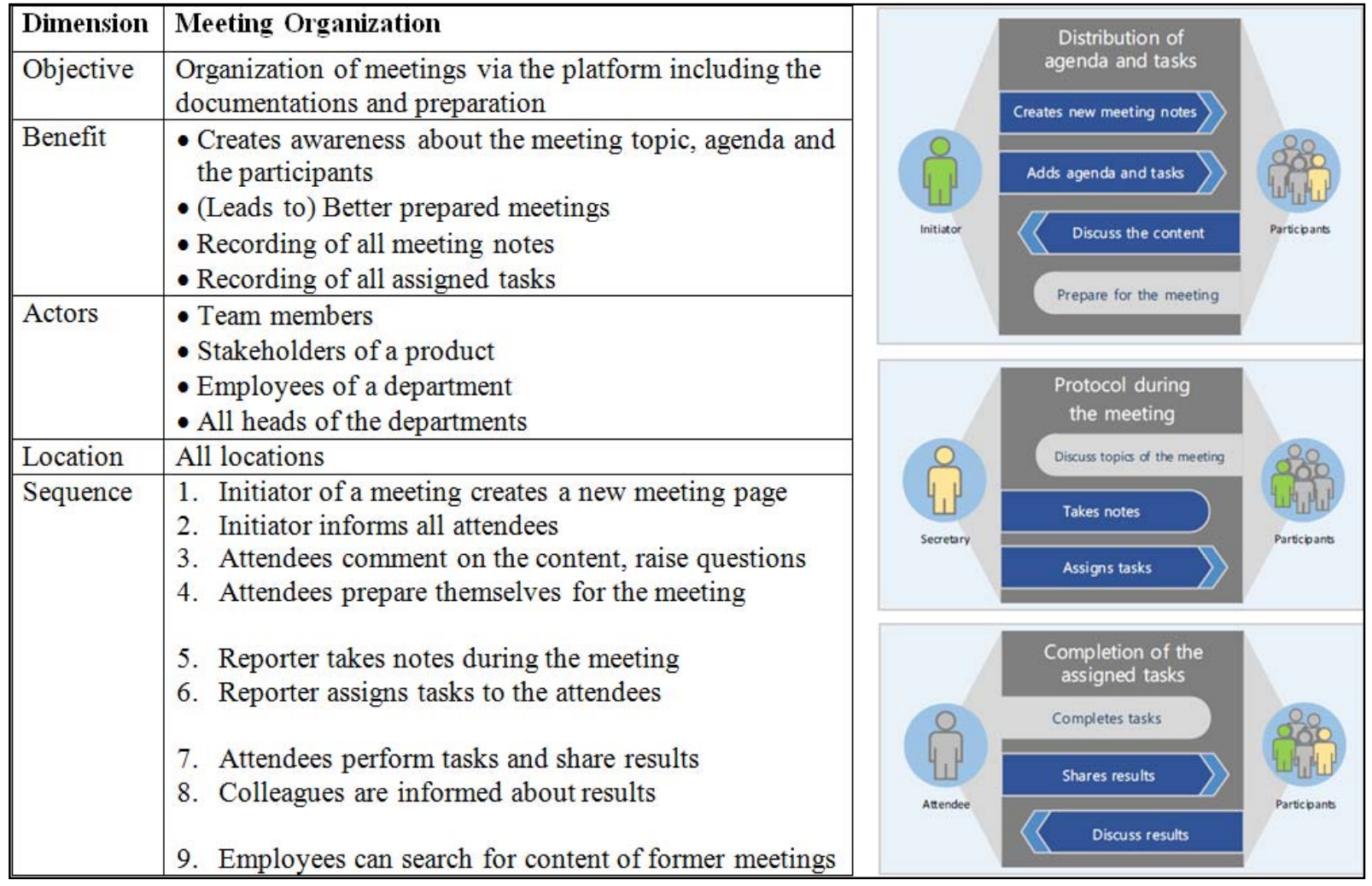

Figure 2: Expository instantiation of the "meeting organization" use case (left: textual description; right: graphical representation) 
objectives

- Actors: Number and type of employees that are incorporated into the use case. It provides an orientation as to the roles that have to be considered

- Location: Listing of all incorporated locations. It provides hints about cultures and time zones that have to be considered

- Sequence: A description of the single actions incorporated into the use case with all specific details

The textual component incorporates the basic information that cannot, or can hardly, be transformed into a graphic.

The graphical representations of the use cases consist of the initiators, the co-actors, the various actions and sub-actions, as well as the direction of the action between the two parties (cf. Fig 2. righthand column).

All use cases are open to every user. If a group wants to restrict the permissions of specific content (e.g. the meeting notes), this is an individual decision that does not depend on the actual use case. The colored differentiation of the actors makes the model more clear and intuitive for the viewer of the graphic. Moreover, it can be derived to which group a certain role belongs to. Furthermore, actions that are addressing other persons (e.g. share results) are marked with an arrow. Those that take place "inside" the actors(s) and do not directly address the other party have a rounded end (e.g. take notes). Actions that take place outside of the platform but are necessary to understand the use case are marked with a gray instead of a blue background (e.g. prepare for the meeting).

For the design and introduction of the ESS use cases, key users or heads of department, as well as management, should be involved. This leads to a higher degree of acceptance and ensures the adoption of the use cases resulting in a higher usage of the platform. However, it is important that the number of prescribed use cases and guidelines are in balance with the free and open use, so that enough utilization room remains [36]. Therefore, it is important that the selection of use cases apply not only to an isolated group of users but preferably involve a plurality of different stakeholders. Through the transmitted network effect [42], the entire ESS appropriation is promoted further. With international collaboration it is important to define a common language and support it in the use case.

This way of depicting use cases has plenty of advantages for the stakeholders. For example, while modeling a scenario, critical bottlenecks or process barriers will be identified that were not visible before. An even more important benefit of this representation is that various new interrogations will arise, once it comes to the detailed definition of single actions and their surroundings. Such issues will not emerge when a use case is examined only on the basis of the functions of the ESS rather than real business cases. The resulting problems can lead to misconceptions or misconstructions of platforms that do not align with the requirements of the users.

After a reasonable time, the use cases should be evaluated to check whether the original aims and requirements are still being met, whether there are technical innovations that support the use case better, and whether other barriers exist that require a modification of the ESS use cases.

\section{Conclusion}

ESS can be characterized as malleable end-user software, i.e. it does not lend itself to immediate forms of usage determined or prescribed by its features [37]. In other words, ESS has no clearly a priori defined use cases. Consequently, it is hard to predict how and in what form ESS will be put to use when rolled out to a particular organizational context. This makes it difficult for platform managers to introduce ESS and facilitate their appropriation.

The study presented here sheds light on the design options for use cases in order to support the ESS appropriation and guide the users. Based on a rich data set consisting of interviews with 10 department heads, usage observations, and a survey at a medium-sized German service provider, we suggest a conceptual model intended as decision support during the design phase of ESS use cases. We created five expository instantiations in the form of ESS use cases at the case company EQS. We found that (1) the involvement of the users and management in the design process, (2) the integration of the use cases in daily work practices, and (3) the inclusion of a wide range of stakeholders as actors of the use cases represent key success factors.

In summary, we have provided a practice-oriented tool for the support of ESS appropriation. Our design theory is intended to give practitioners as well as researchers a scope of decision-making regarding the design and introduction of ESS use cases.

A limitation of the study is that only one case company was examined, and companies in other industries might focus on different use cases. Although we cannot claim that our study is representative, its explorative character nevertheless produced results that we want to extend further in future research. Finally, in order to better validate the proposed model, we are in the process of collecting more datasets from other cases. 


\section{Acknowledgements}

The authors would like to thank the Editor, and four anonymous reviewers for their excellent comments and suggestions. We want to thank also Sebastian Behrendt and Raffaele Fabio Ciriello for providing helpful comments on an earlier version of this manuscript.

\section{References}

[1] R. Agarwal, A.K. Gupta, and R. Kraut, "Editorial Overview - The Interplay Between Digital and Social Networks," Information Systems Research, 19(3), 2008, pp. 243-252.

[2] S. Aral, C. Dellarocas, and D. Godes, "Introduction to the Special Issue - Social Media and Business Transformation: A Framework for Research," Information Systems Research (24:1), 2013, pp. 3-13.

[3] R. Baskerville, and J. Pries-Heje, "Explanatory Design Theory," Business \& Information Systems Engineering (52:5), 2010, pp. 259-271.

[4] A. Bryman, and E. Bell, Business Research methods, Oxford University Press, New York, 2007.

[5] M. Chui, J. Manyika, J. Bughin, R. Dobbs, C. Roxburgh, H. Sarrazin, G. Sands, and M. Westergren, "The social economy: Unlocking Value and Productivity Through Social Technologies,” McKinsey Global Institute Report, 2012.

[6] J.M. DiMicco, D.R. Millen, W. Geyer, C. Dugan, B. Brownholtz, and M. Muller, "Motivations for social networking at work," Proceedings of the Conference on Computer Supported Cooperative Work, San Diego, 2008.

[7] P. Dourish, "The Appropriation of Interactive Technologies: Some Lessons from Placeless Documents,“ Computer Supported Cooperative Work (12:4), 2003, pp. 465-490.

[8] H.S. Du, and C. Wagner, "Weblog Success: Exploring the Role of Technology," International Journal of HumanComputer Studies (64:9), 2006, pp. 789-798.

[9] L. Dubé, and G. Paré, "Rigor in Information Systems Positivist Case Research: Current Practices, Trends, and Recommendations," MIS Quarterly (27:4), 2003, pp. 597636.

[10] R. Dubin, Theory building, The Free Press, New York, 1978.

[11] R.F. Easley, S. Devaraj, and J.M. Crant, "Relating Collaborative Technology Use to Teamwork Quality and Performance: An Empirical Analysis," Journal of Management Information Systems (19:4), 2003, pp. 247-268.
[12] L. Efimova, and J. Grudin, "Crossing Boundaries: A Case Study of Employee Blogging," Proceedings of HICSS, 2007, pp. 86-86.

[13] K.M. Eisenhardt, and M.E. Graebner, "Theory Building from Cases: Opportunities and challenges", Academy of Management Journal (50:1), 2007, pp. 25-32.

[14] EQS Group AG, 3-Monatsbericht 2015 der EQS Group AG, Munich, May 2015.

[15] S. Faraj, S.L. Jarvenpaa, and A. Majchrzak, "Knowledge collaboration in online communities," Organization Science (22:5), 2011, pp. 1224-1239.

[16] G.G. Gable, "Integrating Case Study and Survey Research Methods: An Example in Information Systems," European Journal of Information Systems (3:2), 1994, pp. 112-126.

[17] M. Gibbert, W. Ruigrok, and B. Wick, "What passes as a rigorous case study?" Strategic Management Journal, 29 (13), 2008, pp. 1465-1474.

[18] S. Gregor, and D.S. Hovorka, "Causality: The elephant in the room in information systems epistemology," Proceedings of the European Conference on Information Systems (ECIS), Helsinki, Finland, 2011.

[19] S. Gregor, and D. Jones, "The Anatomy of a Design Theory," Journal of the Association for Information Systems (8:5), 2007, pp. 312-335.

[20] S. Gregor, O. Müller, and S. Seidel, "Reflection, Abstraction, and Theorizing in Design and Development Research," Proceedings of the European Conference on Information Systems (ECIS), Utrecht, Netherlands, 2013.

[21] R.W. Gregory, and J. Muntermann, "Heuristic Theorizing: Proactively Generating Design Theories," Information Systems Research (25:3), 2014, pp. 639-653.

[22] A.R. Hevner, S.T. March, J. Park, and S. Ram, "Design Science in Information Systems Research," MIS Quarterly (28:1), 2004, pp. 75-105.

[23] C. Herzog, A. Richter, M. Steinhüser, U. Hoppe, and M. Koch, "Methods and metrics for measuring the success of Enterprise Social Software - what we can learn from practice and vice versa," Proceedings of the European Conference on Information Systems, Utrecht, Netherlands, 2013.

[24] I. Jacobson, M. Christerson, P. Jonsson, and G. Vergaard, "Object-Oriented Software Engineering, A Use Case Driven Approach," Addison-Wesley, Wokingham, 1992.

[25] G.C. Kane, M. Alavi, G. Labianca, and S.P. Borgatti, "What's Different About Social Media Networks?: A 
Framework and Research Agenda," MIS Quarterly, 38(1), 2014, pp. 275-304.

[26] M. Koch, "CSCW and Enterprise 2.0 - Towards an Integrated Perspective,” BLED Proceedings, 2008, paper 15.

[27] M. Kügler, S. Smolnik, and P. Raeth, "Determining the Factors Influencing Enterprise Social Software Usage: Development of a Measurement Instrument for Empirical Assessment," Proceedings of HICSS, 2013, pp. 3635-3644.

[28] J. S. Lee, J. Pries-Jeje, and R. Baskerville, "Theorizing in design science research," Proceedings of the International Conference on Design Science Resarch in Information Systems and Technology (DESRIST), 2011.

[29] S.T. March, and G.F. Smith, "Design and natural science research on information technology," Decision Support Systems (15:4), 1995, pp. $251-266$.

[30] W. J. Orlikowski, and C.S. Iacono, "The truth is not out there: An enacted view of the Digital Economy," Understanding the Digital Economy, MIT Press, London.

[31] K. Peffers, T. Tuunanen, M. Rothenberger, and S. Chatterjee, "A design science research methodology for information systems research," Journal of Management Information Systems (24:3), 2007, pp. 45-77.

[32] P. Raeth, M. Kügler, and S. Smolnik, "The Impact of Organizational Social Web Site Usage on Work Performance: A Multilevel Structural Interaction Perspective," Proceedings of HICSS, 2012, pp. 4011-4020

[33] P. Raeth, N. Urbach, S. Smolnik, and B. Butler, "Corporate Adoption of Social Computing: A Process-Based Analysis," Journal of Information Technology Case \& Application Research (14:2), 2012, pp. 3-27.

[34] E.M. Rogers, Diffusion of Innovations, The Free Press, New York, USA, 1995.

[35] A. Richter, and K. Riemer, "Corporate Social Networking Sites - Modes of Use and Appropriation through Co-Evolution," Australasian Conference on Information Systems, 2009.

[36] A. Richter, and K. Riemer, "The Contextual Nature Of Enterprise Social Networking: A Multi Case Study Comparison," Proceedings of the 21st European Conference on Information Systems (ECIS), Utrecht, Netherlands, 2013.

[37] A. Richter. K, Riemer, "Malleable End-User Software," Business \& Information Systems Engineering, 2013.
[38] A. Richter, A. Stocker, S. Müller, and G. Avram "Knowledge management goals revisited," VINE, (43:2), 2013 , pp. $132-148$.

[39] H-G. Ridder, "The Theoretical Contribution of Case Study Research to the Field of Strategy and Management," Research methodology in strategy and management (5), 2009, pp. 137-175.

[40] K. Riemer, P. Overfeld, P. Scifleet, and A. Richter, "Oh, SNEP! The Dynamics of Social Network Emergence the case of Capgemini Yammer," Business Information Systems Working Paper, Sydney, Australia, 2012.

[41] A. Stocker, A. Richter, P. Hoefler, and K. Tochtermann, "Exploring appropriation of enterprise wikis: A multiple-case study," Computer Supported Cooperative Work (21:2-3), 2012, pp. 317-356.

[42] C. Shapiro, and H.R. Varian, Information Rules. Havard Business School Press, 1999.

[43] H. Simon, The Sciences of the Artificial, 3rd edition, MA: MIT Press, Cambridge, 1996.

[44] L. Spencer, J. Ritchie, J. Lewis, and J. Dillon, J., "Quality in Qualitative Evaluation: A Framework for Assessing Research Evidence," National Centre for Social Research, Government Chief Social Researcher's Office, London, 2003.

[45] U. Schultze., and M. Avital, "Designing Interviews to Generate Rich Data for Information Systems Research," Information and Organization (21:1), 2011, pp. 1-16.

[46] D. Tilson, K. Lyytinen, and C. Soerensen, "Digital infrastructures: The missing IS research agenda," Information Systems Research , 21 (4), 748-759.

[47] E.W.K., Tsang, "Case studies and generalization in information systems research: A critical realist perspective," Journal of Strategic Information Systems (23:2), 2014, pp. 174-186.

[48] H. van der Heijden, "User Acceptance of Hedonic Information Systems," MIS Quarterly (28:4), 2004, pp. 695704.

[49] V. Venkatesh, S.A. Brown, H. and Bala, "Bridging the Qualitative-Quantitative Divide: Guidelines for Conducting Mixed Methods Research in Information Systems," MIS Quarterly (37:1), 2013, pp. 21-54.

[50] J.G. Walls, G.R. Widemeyer, and O.A. El Sawy, "Building an Information System Design theory for Vigilant EIS," Information Systems Research (3:1), 1992, pp. 36-59. 